\title{
Tight Binding Hamiltonians and Quantum Turing Machines
}

\author{
Paul Benioff \\ Physics Division, Argonne National Laboratory \\ Argonne, IL 60439 \\ e-mail: pbenioff@anl.gov \\ (May 29, 2022)
}

\begin{abstract}
This paper extends work done to date on quantum computation by association of potentials with different types of steps. Quantum Turing machine Hamiltonians, generalized to include potentials, correspond to sums over tight binding Hamiltonians each with a different potential distribution. Which distribution applies is determined by the intitial state. An example, which enumerates the integers in succession as binary strings, is analyzed. It is seen that for some initial states, the potential distributions have quasicrystalline properties and are similar to a substitution sequence.
\end{abstract}

89.70.+c, $71.23 \mathrm{Ft}$

\section{INTRODUCTION}

Quantum computation is a field of much interest. Since the early work [1,2], much impetus was provided by Shor's discovery [3] that for some problems quantum computers are more efficient than classical computers. Recent work includes discussion of quantum error correction [4], use of quantum gates for computation [5], and Hamiltonian descriptions of quantum Turing machines [6, (7).

In all work to date models of quantum computation are considered in which successive steps of the computation proceed smoothly with no potentials present, if the system is isolated from the environment (see for example [8]). Landauer [9] has noted that environmental influences and defects in physical models introduce random potentials which degrade performance by causing reflections at various steps and decay of the transmitted component.

Here potentials are introduced into quantum computation, not in a random fashion, but by association with different types of steps. An example is the association of a potential with all Turing machine "read 1" steps. All other steps are potential free.

The method used is to generalize the description of quantum Turing machines [1, 7] to include potentials at one or more types of computation steps. Isolation from the environment is assumed. In this case the Feynman [17] Hamiltonian for these generalized quantum Turing machines (GQTM)s, can be also be described as a sum of tight binding Hamiltonians each of which has a different potential distribution along a one dimensional path of computation states. Which of these Hamiltonians applies to the GQTM is determined by the initial state.

For some GQTMs the potential distributions are similar to those which are of much recent interest in condensed matter physics. This includes Hamiltonians with quasiperiodic potentials [10] or with potential distributions corresponding to substitution sequences (see 11] for a definition). Examples include the Period Doubling, [12,13], Thue- Morse 12,13], Fibonacci [14, and RudinShapiro [15] substitution sequences. This similarity provides a link between the behavior of a GQTM as it evolves during a computation and the 1-D motion of a particle such as an electron in systems with potentials which are quasiperiodic or correspond to substitution sequences.

This similarity will be shown here by first giving a brief description of the physical model and the sum decomposition of the GQTM Hamiltonian. An example, the counting GQTM which enumerates the nonnegative integers in succession as binary strings will be considered. This is of interest because some of the potential distributions are similar to but not identical with those for both quasicrystals and substitution sequences. The differences suggest that the distributions are a new type of 1-D structure that does not seem to have been discussed in the literature.

\section{THE PHYSICAL MODEL}

For quantum Turing machines, the physical model corresponds to one tape machines. It includes a finite state head that moves along an infinite lattice of finite state systems called qubits. For many computations it is sufficient to consider binary strings corresponding to qubit states $|0\rangle,|1\rangle$. The actual physical form of each qubit (e.g. as a spin 1/2 system, etc.) is not important here.

The computation basis $B$ which spans a separable Hilbert space $\mathcal{H}$ is the set $\{|l, j, S\rangle\}$ of states. Here $j$ and $l$ denote the lattice location and internal state of the head. $|S\rangle=\otimes_{j=-\infty}^{\infty}|S(j)\rangle$ denotes the lattice qubit state where $S$ is any function from the integers into the set of possible qubit states such that $S(j) \neq 0$ for at most a finite number of $j$ values. This condition, the 0 tail state condition, is one of many that can be imposed to keep $B$ denumerable. Both $B$ and $\mathcal{H}$ depend on the condition imposed. 


\section{STEP OPERATORS AND HAMILTONIANS}

Each GQTM is described by a step operator $T$ such that iteration of $T$ or its adjoint corresponds to successive steps of the GQTM in the forward or backward time direction. $T$ is defined as a finite sum of elementary step operators, $T=\sum_{l, s} T_{l, s}=\sum_{l, s} \gamma_{l, s} W_{l, s}$, over head states $l$ and qubit states $s$ where $\gamma_{l, s}$ is a real constant between 0 and $1 . . W_{l, s}$ has the form,

$$
W_{l, s}=\sum_{j=-\infty}^{\infty} w_{l, s} Q_{l} v_{l, s} P_{s, j} u_{l, s} P_{j}
$$

where $Q_{l}, P_{j}, P_{s, j}$ are respective projection operators for the head in internal state $|l\rangle$, at site $j$, and the site $j$ qubit in state $|s\rangle$.

The unitary operators $w_{l, s}, v_{l, s}, u_{l, s}$ describe head and qubit state changes, and head motion. The dependence on $l, s$ is indicated by the subscripts. The operators $w_{l, s}$ and $u_{l, s}$ satisfy the commutation relations, $w_{l, s} Q_{l}=Q_{l^{\prime}} w_{l, s}$ for some $l^{\prime} \epsilon L$ and $u_{l, s} P_{j}=P_{j \pm 1} u_{l, s}$. Here $L$ is the finite set of head states and $P_{j+1}, P_{j-1}$ correspond to head motion one site to the right or one site to the left. For our purposes it is sufficient to require that $v_{l, s}$ satisfy $v_{l, s} P_{s, j}=P_{s^{\prime}, j} v_{l, s}$ where $s^{\prime} \epsilon \mathcal{S}$, the set of qubit states. If the qubits are binary this is equivalent to requiring that $v_{l, s}=\sigma_{x}$ or $v_{l, s}=1$.

These step operators generalize earlier definitions [1, 6, 7] in that values of $\gamma_{l, s}$ different from 0 or 1 are allowed. Potentials are associated with steps for which terms $T_{l, s}$ with $\gamma_{l, s}<1$ are active.

The main condition imposed on $T$ is that it be distinct path generating on the computation basis $B$. This condition can be relaxed to apply to any basis, not just $B$ (see [7] for details) but this generality is not needed here. The condition means that iteration of $T$ or $T^{\dagger}$ on the states in $B$ generates finite or infinite paths of states which do not join, branch, or intersect. Mathematically this is expressed by requiring that $T$ be a direct sum of weighted shifts [16]. That is $T=U D=\sum_{i} U_{i} D_{i}$ where for each $i U_{i}$ is a bilateral shift, a unilateral shift, the adjoint of a unilateral shift, a finite truncated shift of length $N$, or a cyclic shift of length $M$ with $N, M$ arbitrary. $D$ is defined by $D|l, j, S\rangle=\gamma_{l, S(j)}|l, j, S\rangle$ for any state $|l, j, S\rangle$ in $B$ not annihilated by $U$ or $U^{\dagger}$. Both $U$ and $D$ satisfy $U=\sum_{i} U_{i} P_{i}, D=\sum_{i} D_{i} P_{i}$ where the $P_{i}$ are orthogonal multidimensional projection operators on $\mathcal{H}$.

The $i$ sum is a sum over paths where each path $i$ in $B$ is defined by iteration of $U_{i}$ or $U_{i}^{\dagger}$. That is if the state $|l, j, S\rangle$ is not annihilated by $U_{i}$ or $U_{i}^{\dagger}$ then path $i$ is defined by $\cdots U_{i}^{\dagger}|l, j, S\rangle,|l, j, S\rangle, U|l, j, S\rangle, \cdots$.

For each step operator $T$ define a Hamiltonian by [17],

$$
H=K\left(2-T-T^{\dagger}\right)
$$

where $K$ is an arbitrary constant. If $T=U D$, then $H$ can also be written as $H=\sum_{i} H_{i} P_{i}$ where $H_{i}=$
$K\left(2-T_{i}-T_{i}^{\dagger}\right)$ describes motion along the path states in $B_{i}$. Eq.2 is based on the association of an infinitesimal time interval with $T$ so that it can be directly used to construct a Hamiltonian.

From this it follows that for each path $i, H_{i}$ is a tight binding Hamiltonian on $P_{i} \mathcal{H}$ with nearest neighbor off diagonal potentials. To see this note that

$$
H_{i}=K\left(2-U_{i}-U_{i}^{\dagger}\right)+V_{i}
$$

where the off-diagonal potential $V_{i}$ along path $i$ is given by $V_{i}=K\left[U_{i}\left(1-D_{i}\right)+\left(1-D_{i}\right) U_{i}^{\dagger}\right]$. The constant term $2 K$ is present so that for each path, the path kinetic energy component of $H_{i}, K\left(2-U_{i}-U_{i}^{\dagger}\right)$ is equivalent to the symmetrized discrete version of the second derivative, $-K d^{2} /(d x)^{2}$.

Under the action of $H$ the initial state $\Psi(0)$ determines which tight binding Hamiltonian applies. If $\Psi(0)$ is a linear sum of computation states in $P_{i} \mathcal{H}$, then $H_{i}$ gives the evolution of $\Psi(0)$. If $\Psi(0)$ has components in more than one subspace $P_{i} \mathcal{H}$, then each component has a corresponding $H_{i}$ which describes its evolution.

For many $i$ the potential distributions in the corresponding $H_{i}$ are the same. This is the case for any transformation group whose actions do not change $T$. For example, for GQTMs, each shift of an initial state along the lattice corresponds to a different path $i$ with the same $H_{i}$. From this one sees that equivalence classes $\mathcal{P}_{i}=\left\{P_{j}: H_{j}=H_{i}\right\}$ of projectors can be defined which identify paths with the same $H_{i}$.

It is important to distinguish between path motion and head motion on the qubit lattice. In general, iterations of $T$ describe head motion backward and forward over lattice sites. However, (cyclic paths excepted) for each passage over a site, the overall system state is orthogonal to the state for any other passage. As a result the back and forth lattice motion can be unfolded to motion along a path where the path states are pairwise orthogonal and are ordered by iteration of $T$ or $T^{\dagger}$. The tight binding Hamiltonians, Eq. 3, refer to motion along paths and not the qubit lattice.

\section{THE COUNTING GQTM}

Simple examples can be constructed by letting $\gamma_{l, s}$ assume just two values, 1 and $\gamma$ where $\gamma$ is a real number between 0 and 1. In particular, for all $l \epsilon L$, let $\gamma_{l, 1}=\gamma$ and $\gamma_{l, s}=1$ for all $s \neq 1$.

This simplification corresponds to GQTMs in which a potential is associated with all "read 1" elementary steps (those steps with $P_{s j}=P_{1 j}$ in Eq. 11). All other qubit state read actions are potential free. In this case the $l, s$ sum for $T$ can be split into two parts, $T=\sum_{l, s \neq 1} W_{l, s}+$ $\gamma \sum_{l} W_{l, 1}$.

The counting GQTM can be used to illustrate the foregoing. This GQTM, starting with a blank qubit lattice 
(all $0 s$ ) with two markers spaced $\mathrm{n}+1$ sites apart, generates all binary numbers in succession from 0 up to $2^{n}-1$ by continually adding 1 .

The step operator for this GQTM, which is used in Eq. 2, is a sum of 7 terms:

$$
\begin{aligned}
T= & \sum_{j=-\infty}^{\infty}\left(Q_{0} P_{0 j} u P_{j}+w Q_{0} P_{2 j} u P_{j}+Q_{1} P_{0 j} u P_{j}\right. \\
& +w Q_{1} P_{2 j} u^{\dagger} P_{j}+\gamma Q_{2} v_{x j} P_{1 j} u^{\dagger} P_{j}+w^{\dagger} Q_{2} v_{x j} P_{0 j} u P_{j} \\
& \left.+w Q_{2} P_{2 j} u P_{j}\right)
\end{aligned}
$$

The projection operators are as defined for Eq. 1; $w$ is a shift mod 3 on the three head states $\left(w Q_{m}=\right.$ $\left.Q_{m+1} w \bmod 3\right)$ and $u$ shifts the head along the lattice by one site $\left(u P_{j}=P_{j+1} u\right)$. The need for markers is accounted for here by choosing the qubits in the lattice to be ternary with states $|0\rangle,|1\rangle,|2\rangle .|2\rangle$ is used as a marker and $|0\rangle,|1\rangle$ are used for binary strings. The qubit transformation operator for the site $j$ qubit $v_{x j}=\sigma_{x j}\left(P_{0 j}+P_{1 j}\right)+P_{2 j}$ exchanges the states $|0\rangle,|1\rangle$ and leaves the state $|2\rangle$ alone.

The terms in $T$ are chosen so that $T$ is distinct path generating on $B$. The theorems given in [7], that verify this conclusion were proved for $D=1$. The proofs should also hold for $D \neq 1$.

An initial state for this GQTM is shown in Figure 1 with the head in state $|0\rangle$ in a wave packet localized to the left of the origin. All qubits are in state $|0\rangle$ except those at sites $0, n+1$ which are in state $|2\rangle$. Successive iterations of $T$ move the head to the righthand units marker followed by successive enumeration of the first $2^{n}$ numbers as binary strings. When the space between the markers is filled with $1 s$, the initial state is restored by conversion of the $1 s$ to $0 s$. The head in state $|1\rangle$ moves to the right with no more qubit or head state changes.

For this GQTM, a potential of height $V=2 K(1-\gamma)$ is present at path states at which term 5 is active. This is the only term with a "read 1" operator. The path potential distribution is determined by a close examination of successive iterations of $T$. For example, to add 1 to $\cdots 01001112$ to obtain $\cdots 01010002$ with the head beginning and ending in state $|1\rangle$ at the units marker 2 requires one "read 2" step (term 4) followed by three "read 1 " plus $1 \rightarrow 0$ steps (term 5 ) followed by one "read 0 " plus $0 \rightarrow 1$ step (term 6 ) followed by three "read 0 " steps (term 3 ). In this path segment there is a potential of height $V$ and width 3 with potential free regions of width 1 to the left and width 4 to the right. Extension of the segment shows a few more potential free sites in both directions before encountering the next potential.

The path potential distribution is expressed in general as follows: Let $R$ be a function from the nonnegative integers to the nonnegative integers such that $R(j)$ gives the number of $1 s$ occurring before the first 0 in the binary string for $j . R(j)=0$ if $j$ even and $R(1)=1, R(3)=$
$2, R(5)=1, R(7)=3, \cdots . R$ can be expressed recursively by

$$
R_{n}=S_{n-1} n ; \quad S_{n}=R_{n} S_{n-1}
$$

for $n=1,2, \cdots$ with $S_{0}=0$ where $R_{n}$ is the initial segment of $R$ of length $2^{n}$.

It turns out that $R$ is a substitution sequence 11] with the substitution rule $n \rightarrow 0 n+1$ for $n=0,1,2, \cdots$. (That is, the infinite sequence $0,1,0,2,0,1,0,3, \cdots$ is invariant under the replacement of $n$ with $0 n+1$.) This generalizes the literature definition of substitution sequences [13, 18, 19 in that the alphabet is infinite.

A study of the iteration of $T$ with the initial head state $|1, n+1\rangle$ and $|S\rangle$ as shown in Fig. 1 shows that the potential distribution is given by a binary sequence $\mathcal{R}$ obtained from $R$ by replacing $R(j)$ by a string of numbers according to the following prescription: $0 \rightarrow 00=\underline{0}$ and $n \rightarrow 0 n 0^{n+1}=\underline{n}$. Here $0^{n+1}$ denotes a string of $\bar{n}+1$ $0 s$ and $n$ denotes a string of $n 1 s$. Justification for this can be seen by extension of the example of adding 1 to …01001112.

$\mathcal{R}$ is generated recursively by Eqs. 5 if $n$ is replaced by $\underline{n}$ and $S_{0}=\underline{0}$. It follows that $\mathcal{R}$ is also a substitution sequence for the words $\underline{0}, \underline{1}, \cdots$ regarded as an alphabet. The recursive generation is important for future work on the spectral and transmission properties of tight binding Hamiltonians with this potential distribution.

The above is illustrated in Figure 2 for $n=6$. The figure shows the distribution of read 0 and read 2 steps (as $0 s$ ) and read 1 steps (as $1 s$ ) as a function of the number of $T$ iterations starting from the initial head state $|1, n+1\rangle$ and the two markers separated by 6 sites (Figure 1). Fig. 2 also gives the potential distribution for enumerating the first 64 numbers as binary strings. Changing the initial state to any component of the wave packet shown in Figure 1 shifts the distribution along the path with no changes in the width or spacing between the potentials.

Different initial states correspond to different potential distributions. Changing the separation $n+1$ between the two markers changes the number of potentials present. Initial states with $m$ markers separated by $n_{1}+1, n_{2}+1, \ldots, n_{m}+1$ qubit sites give a potential distribution obtained by concatenating (with some intervening $0 s$ ) initial segments of $\mathcal{R}$ corresponding to enumeration of the first $2^{n_{1}}, 2^{n_{2}}, \ldots, 2^{n_{m}}$ numbers. If all the $n_{i}=p$ are the same, the potential distribution is periodic with $m$ periods each containing the first $2^{p-1}$ potentials.

If just one marker is present and the head is in state $|1\rangle$, then all binary numbers are generated in succession without halting. For this input state the potential distribution in $H_{i}$, Eq. 3, is given by $\mathcal{R}$. The system is quasicrystalline in that no two path sites have the same global potential environment, yet each local environment of arbitrary length in $\mathcal{R}$ is repeated infinitely often [20]. 
Differences between this distribution and those described in the literature include the facts that the potential distribution is one-way infinite, the alphabet is infinite, and $\mathcal{R}$ is a substitution sequence with respect to alphabet words and not the original alphabet. Because of this the 1-D structure appears to be of a new type not yet analyzed.

\section{ACKNOWLEDGEMENTS}

This work is supported by the U.S. Department of Energy, Nuclear Physics Division, under contract W-31-109ENG-38.

[1] P. Benioff, Jour. Stat. Phys. 22563 (1980).

[2] D. Deutsch, Proc. Roy. Soc. London A 400997 (1985).

[3] P. Shor, in Proceedings of the 35th Annual Symposium on the Foundations of Computer Science (IEEE Computer Society, Los Alamitos, CA 1994), p. 124. For a recent review see A. Ekert and R. Jozsa, Rev. Mod. Phys. 68 733 (1996).

[4] P. W. Shor, Phys. Rev. A 52 R2493 (1990); R. LaFlamme, C. Miquel, J.P. Paz, and W. H. Zurek, Phys. Rev. Letters 77198 (1996).

[5] S. Lloyd, Phys. Rev. Letters, bf 75346 (1995); D. P. DiVencenzo, Phys. Rev. A 511015 (1995); D. Deutsch, A. Barenco, and A. Ekert, Proc. Roy. Soc. London A 449 669 (1995).

[6] P. Benioff Ann. NY Acad. Sci. 480475 (1986).

[7] P. Benioff, Phys. Rev. A 541106 (1996).

[8] S. Lloyd, Jour. Modern Optics, 412503 (1994).

[9] R. Landauer, Physics Letters A 217188 (1996); Philos. Trans. Roy. Soc. London Ser. A To Appear; Physics Today 4423 (1991); Foundation of Physics 16551 (1986).

[10] The literature is large. See for example D. DiVincenzo and P. Steinhardt Eds., Quasicrystals the State of the Art, Directions in Condensed Matter Physics-Vol. 11, World Scientific, Singapore 1991.

[11] Let $A$ be a finite alphabet, $A^{*}$ the set of all finite words of $A$, and $A^{N}$ the set of all one way infinite words of $A$. A substitution rule replaces each letter $\alpha$ of $A$ with a word from $A^{*}$. For each $\alpha$ a sequence of words in $A^{*}$ can be generated by starting with $\alpha$ and iterating the substitution rule. The limit sequence in $A^{N}$ is a substitution sequence if it is invariant under the rule.

[12] J.M. Luck, Phys. Rev. B 39 5834, (1989); J. Bellissard, A. Bovier, and J-M. Ghez, Commun. Math. Phys. 135 379 (1991).

[13] A. Bovier and J-M. Ghez, J. Phys. A: Math. Gen. 28 2313 (1995).

[14] A. Sütő, Jour Stat. Phys. 56525 (1989).

[15] A. Bovier and J-M. Ghez, Commun. Math. Phys. 15845 (1993); 166431 (1994).

[16] P.R. Halmos, A Hilbert Space Problem Book, 2nd Edition, Springer Verlag, New York, 1982.
[17] R. P. Feynman, Optics News 1111 (1985); reprinted in Foundations of Physics 16507 (1986).

[18] M. Kolář amd F. Nori, Phys. Rev. B 421062 (1990)

[19] M. Queffélec, Substitution Dynamical Systems-Spectral Analysis, Lecture Notes in Mathematics 1294, Editors A. Dold and B. Eckmann, Springer Verlag, Berlin 1987.

[20] D. P. DiVicenzo and P. J. Steinhardt, Progress and Current Issues in Quasicrystals, Reference 9, pp 1-15.

\section{FIGURE CAPTIONS}

Figure 1. Initial and Final States for Counting GQTM for the First $2^{n}$ Binary Numbers. All lattice qubits are in state $|0\rangle$ except those at sites 0 and $n+1$ which are in state $|2\rangle$. The initial and final head states are shown as wave packets with internal head states $|0\rangle$ and $|1\rangle$ to the left and right respectively.

Figure 2. Path Potential Distribution for Enumeration of the first 64 Numbers $(n=6)$ as Binary Strings. The figure shows the distribution of $0 s$, corresponding to read 0 or read 2 steps, and $1 s$, corresponding to read 1 steps, as a function of the number of $T$ iterations starting from the initial head state $|1, n+1\rangle$. 


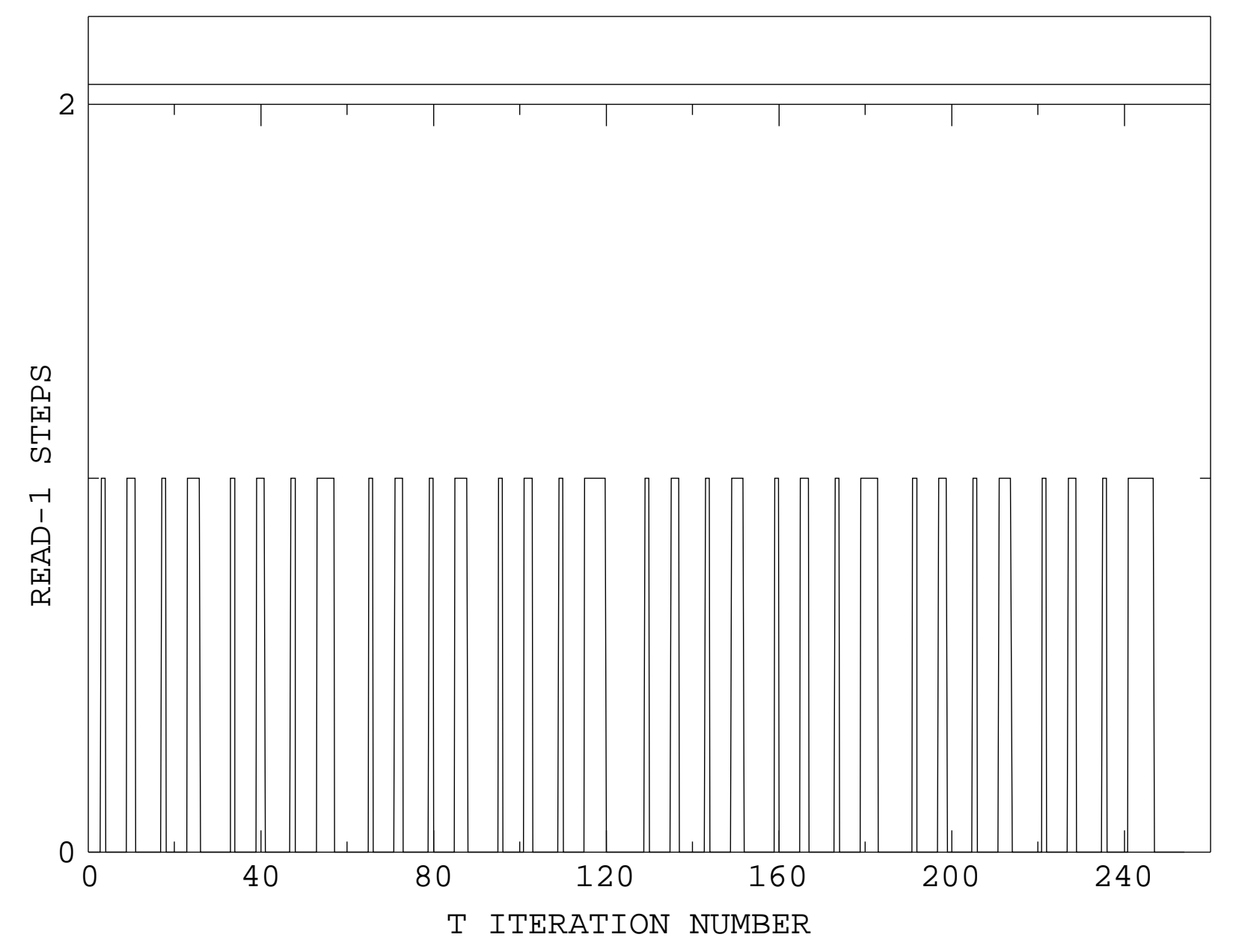

